\title{
Pregnancy in a Secondary Infertile Woman with Polycystic Ovarian Syndrome (PCOS) -A Case Report
}

\author{
Nazeeha Waseem $^{1 *}$, Hafsa Waseem ${ }^{2}$, Beenish Abbas ${ }^{3}$, Rahat Ali $^{4}$, Kiran Aftab ${ }^{5}$
}

${ }^{1}$ Medical Imaging Doctor, Department of Radiological Sciences and Medical Imaging, The University of Lahore, Gujrat, University of Lahore, Adjacent Chenab Bridge, Gujrat, Punjab 50700, Pakistan

${ }^{2}$ Senior Lecturer, Fazaia Medical College, Islamabad, Pakistan

${ }^{3}$ Radiography Technologist, Government College University, Faisalabad, Pakistan

${ }^{4}$ Medical Officer, Holy Family Hospital, Rawalpindi, Pakistan

${ }^{5}$ Lecturer, The University of Lahore, Gujrat, University of Lahore, Adjacent Chenab Bridge, Gujrat, Punjab 50700, Pakistan

DOI: $10.36347 /$ sjmcr.2021.v09i01.019

| Received: 12.01.2021 | Accepted: 23.01.2021 | Published: 26.01.2021

*Corresponding author: Nazeeha Waseem

Abstract

Case Report

Polycystic ovarian syndrome, also called Stein-Leventhal syndrome, is a disorder involving multiple endocrine systems resulting in abnormal levels of gonadotropins, androgens, and glucose in the blood. Several other manifestations arise in association with PCOS like a blockage in the fallopian tube, endometrial polyps, cervical cancer, ovarian cancer, etc. We hereby delineate a case of a 34-year-old married female in whom PCOS was diagnosed at the age of 22. After her marriage, she presented with the complaint of infertility. Investigations revealed that she had a polyp in her right fallopian tube after which a polypectomy was performed. She was able to conceive six months after the surgery was done. Currently, after seven years, she presented with the same complaint of infertility. After undergoing relevant procedures, her secondary infertility was ascertained as a result of PCOS. Following regular medications, she was able to get pregnant with triplets. This pregnancy had many associated complications. Out of three, one fetus died and the remaining two survived.

Keywords: PCOS, Infertility, Metformin, Ovarian Cancer.

Copyright $(\mathcal{C} 2021$ The Author(s): This is an open-access article distributed under the terms of the Creative Commons Attribution 4.0 International License (CC BY-NC 4.0) which permits unrestricted use, distribution, and reproduction in any medium for non-commercial use provided the original author and source are credited.

\section{INTRODUCTION}

The polycystic ovarian syndrome is a multiendocrine disorder occurring in premenstrual women and is on the mountaintop of the factors that cause infertility in women of reproductive age [1]. This syndrome is characterized by oligomenorrhea or sometimes amenorrhea. PCOS causes infertility as well as makes the patient insensitive to insulin. Furthermore, PCOS is not only associated with abnormalities in the reproductive tract but it also causes irregularities throughout the female body [2]. Increase in serum glucose, acne, hirsutism, thinning of hair, androgenic alopecia, thyroid disorders, increase in serum testosterone, and luteinizing hormone, and high blood pressure which can lead to cardiovascular diseases. There is also an increased risk of cancers of the endometrium and cervix in females suffering from PCOS [3]. Obesity, insulin resistance, various toxins including pesticides, bisphenol A (BPA) or phthalates [4], a weakened immune system, poor diet, and family history of PCOS are the major contributors causing this syndrome.

Polycystic ovarian syndrome makes it very difficult for the patient to conceive a child due to abnormal or absent ovulation. The prevalence of infertility is very high in patients suffering from PCOS especially in those who have had a successful delivery in the past. Patients do conceive but only after following a strict medical protocol. Still, there are complications like spontaneous abortions, preeclampsia, impaired glucose tolerance, premature birth, and gestational diabetes that accompany the pregnancy. Patients are advised to avoid a high carbohydrate diet to avoid gestational diabetes otherwise the baby will be overweight. If the baby is a girl, then there is a $50 \%$ chance that she will also have PCOS. Diagnosis of PCOS mainly depends upon very careful clinical assessment [5]. Although some imaging techniques like sonohysterography and hysterosalpingogram and testing the increased level of androgens are there to direct us towards the complete diagnosis but still proper clinical 
assessment is the best help we can get in identifying patients suffering from PCOS.

\section{CASE REPORT}

A 34-year-old married female patient presented with the chief complaint of infertility in Holy Family Hospital, Rawalpindi, Pakistan. She presented with the same complaint 7 years ago two years after getting married. She hadn't conceived before. After taking a complete history, it was discovered that her maternal side of the family had presented with a complaint of polycystic ovarian syndrome. She was a non-smoker and her blood glucose levels revealed that she did not have any diabetes. Her blood pressure was also normal at that time indicating that she was normotensive and there was no abnormality in her complete blood count report either. After doing an ultrasound, it was found that she had a slight increase in the size of the left ovary. As seen in patients with PCOS, the size of the ovaries becomes twice the size of normal ovaries (Figure-1).

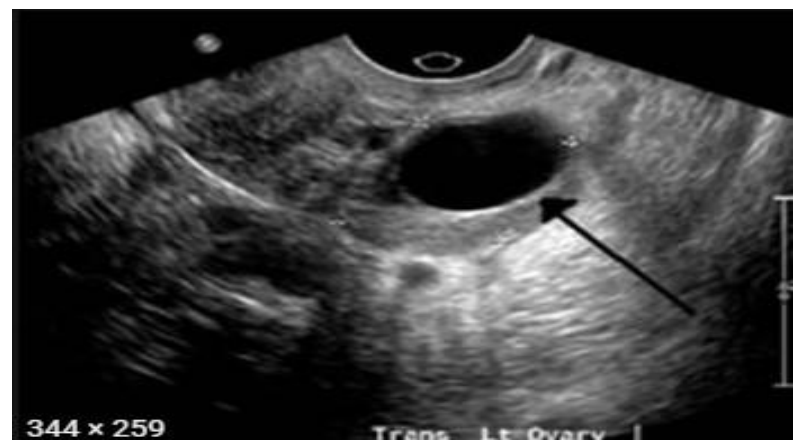

Fig-1: The Ultrasound Image showing, Enlarged Left Ovary

After explaining the procedure to the patient and taking her consent, hysterosalpingography was done. The patient was asked to empty her bladder and placed in a lithotomic position. The cervix was localized and after cleaning with povidone-iodine, a speculum was inserted. $10 \mathrm{ml}$ contrast was given $(3 \mathrm{ml}$ to fill the uterine cavity and $3 \mathrm{ml}$ to fill the fallopian tube) which revealed the presence of polyp in the right fallopian tube (Figure-2). After the surgery, an antibiotic course of doxycycline was prescribed as prophylaxis to prevent any uterine infection.

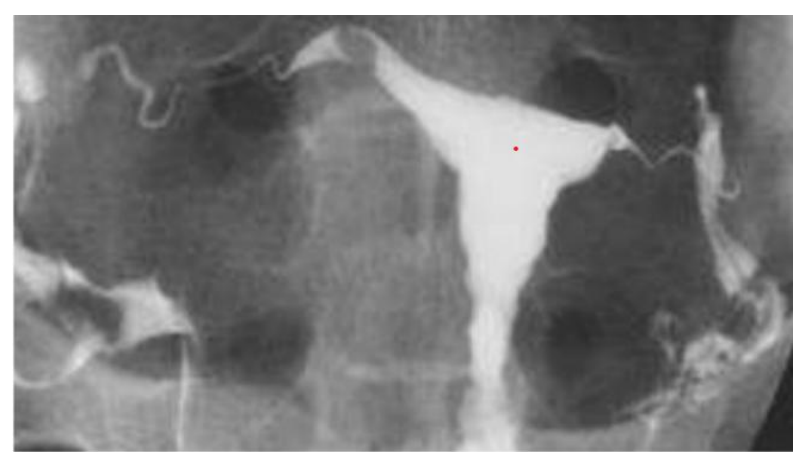

Fig-2: Hysterogram showing Polyp in Right Fallopian tube
Few days after the procedure, surgical removal of the polyp was performed. After polypectomy, this patient was able to conceive after 6 months. Currently, this woman presented with the same complaint of being unable to conceive seven years after her first delivery. In secondary infertility, it is difficult to conceive for a patient who is unable to conceive after a successful delivery. Further imaging revealed tubal patency was normal. Saline infusion sonography was done to check whether the fallopian tubes are open or not. In this case, there was no obstruction and both of the fallopian tubes were patent (Figure $3 \& 4$ ). In secondary infertility, it is difficult to conceive for a patient who is unable to conceive after a successful delivery. Abnormal ovulation, fallopian tube damage, isthmocele, and endometriosis are some of the most common causes of secondary infertility.

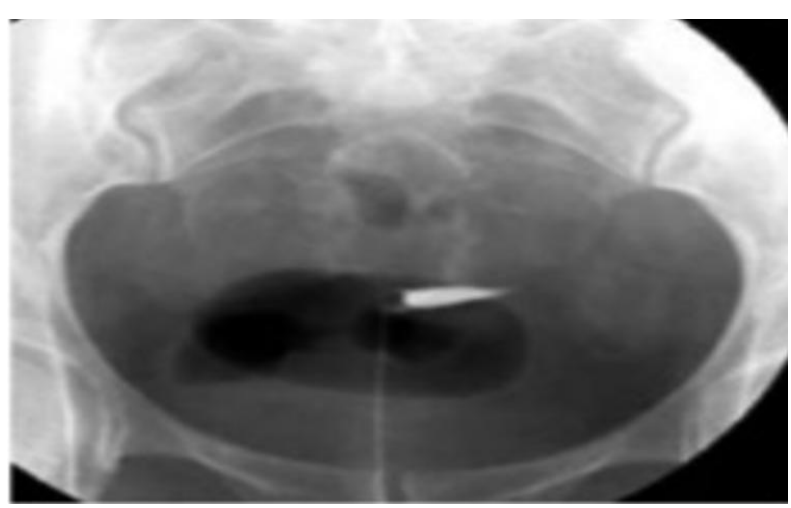

Fig-3: Early filling phase

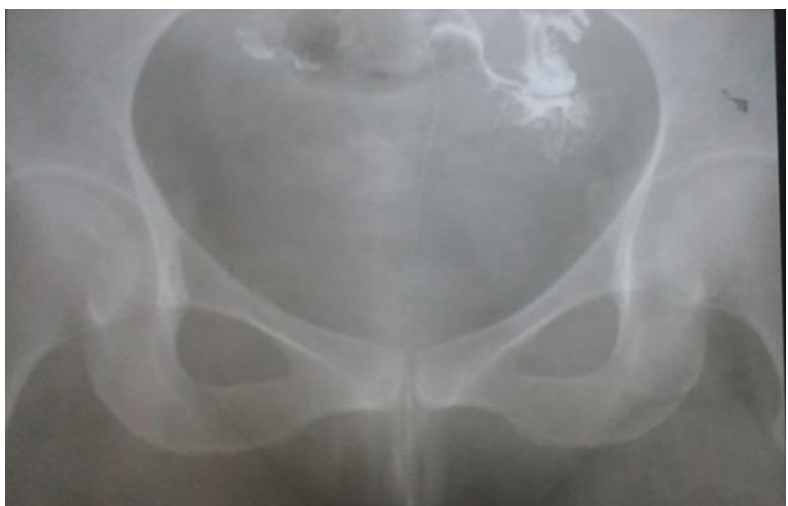

Fig-4: Tube filling phase

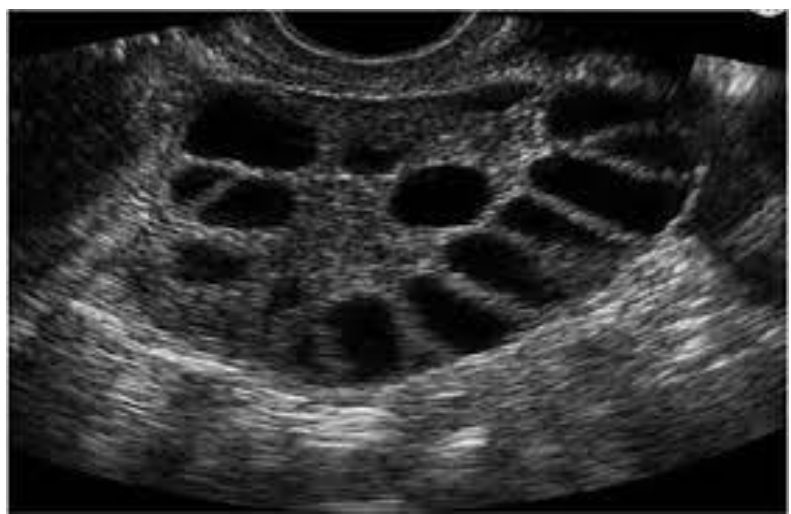

Fig-5: The Ultrasound image clearly revealing Multiple cysts i.e. PCOS 
Following a series of laboratory tests, it was ascertained that secondary infertility in this patient was due to PCOS. (Figure-5) The patient took Lezra $(2.5 \mathrm{mg})$ once a day from the second to the seventh day of her menstrual periods. Lezra is an aromatase inhibitor which blocks the conversion of androgens to estrogen and through a feedback mechanism, increases the concentration of gonadotropins to induce ovulation. Then till the 13th day of her periods, IVF-M injections were given to her. An IVF-M injection contains an equal ratio of FSH: LH. Lastly, an IVF-C injection was given which is a preparation $\mathrm{HCG}$, on the day of ovulation. After sticking to her medication, she was able to conceive again, but this time she was pregnant with female triplets. This pregnancy was nowhere near short of complications. During delivery, two out of three survived while one of them died.

\section{DISCUSSION}

Polycystic ovarian syndrome is one of the commonest of multiple endocrine disorders. It often starts at adolescence and persists throughout the reproductive years of life [6]. Symptoms of PCOS include hair loss, hirsutism, obesity, pelvic pain, overweight, acne, irregular periods, infertility, high testosterone, and high luteinizing hormone and, increased levels of glucose in the blood. Because there is no definitive diagnosis for PCOS other than authentic clinical assessment, it poses serious challenges for clinicians to find an effective treatment of this syndrome [7]. The concentration of luteinizing hormone (LH) increases to that of follicle-stimulating hormone (FSH) and this is the reason for the hyperplasia of theca cells. These theca cells produce androgens mainly testosterone, which is the cause of the development of male secondary sexual characteristics like hirsutism, acne, and deepening of voice [8]. The normal ratio of luteinizing hormone to the follicle-stimulating hormone is 2:1 but in patients suffering from PCOS, this ratio increases sometimes many fold. There are several other disorders associated with PCOS like thyroid and cardiovascular [8]. The microscopic examination of this disease shows hyperplasia of theca cells which takes part in the production of androgens. Theca cells are stimulated by luteinizing hormone (LH) which is released by the anterior pituitary. This hormonal imbalance causes anovulation ultimately resulting in infertility. According to a recent study, the prevalence of primary infertility $(57.5 \%)$ was more than that of secondary infertility (42.5\%) [9]. Infertility may be due to several reasons like a blocked fallopian tube, ovarian cancer, and cervical cancers. Increased prevalence of developing polyps has also been reported. In PCOS, insulin insensitivity and obesity are also noted along with infertility. So these areas are also targeted while establishing a therapeutic approach [10]. Clomiphene is recommended as the primary medication for PCOS [11].

\section{CONCLUSION}

The chances of getting pregnant really take a slope downhill because of the complete anovulation or abnormal ovulation due to hormone imbalance. Secondary infertility in PCOS is also due to hormonal imbalance and even if a patient can conceive, the pregnancy is nothing short of associated complications. The etiology of this syndrome hasn't been completely understood yet and the reason why remains one of the most poorly understood mechanisms amongst the scientific community. Aromatase inhibitors such as letrozole which increases the concentration of FSH to induce ovulation and then IVF-M and IVF-C are given for regulating ovulation. In some cases, metformin is used to target insulin sensitivity and obesity. Cyclic use of progestin is employed to treat menstrual dysregulation. There is also evidence that suggests that controlling metabolic irregularities can help the patient to conceive again.

ACKNOWLEDGMENTS: We thank the patient and her family for allowing us to share her details.

Conflict of Interest: None identified.

CONSENT: All the details mentioned were written after the written consent of patient.

\section{REFERENCES}

1. Patel S. Polycystic ovary syndrome (PCOS), an inflammatory, systemic, lifestyle endocrinopathy. J Steroid Biochem Mol Biol. 2018; 182:27-36.

2. Kowalczyk K, Franik G, Kowalczyk D, Pluta D, Blukacz $Ł$, Madej P. Thyroid disorders in polycystic ovary syndrome. Eur Rev Med Pharmacol Sci. 2017; 21(2):346-60.

3. Hanson B, Johnstone E, Dorais J, Silver B, Peterson CM, Hotaling J. Female infertility, infertility-associated diagnoses, and comorbidities: a review. J Assist Reprod Genet. 2017; 34(2):16777.

4. Rutkowska AZ, Diamanti-Kandarakis E. Polycystic ovary syndrome and environmental toxins. Fertil Steril. 2016; 106(4):948-58.

5. Pasquali R, Gambineri A. A comprehensive approach in diagnosing the polycystic ovary syndrome. Womens Health (Lond Engl). 2015;11(4):501-12

6. Basirat Z, Faramarzi M, Chehrazi M, Amiri M, Ghofrani F, Tajalli Z. Differences between infertile women with and without PCOS in terms of anxiety, coping styles, personality traits, and social adjustment: a case-control study. Arch Gynecol Obstet. 2020; 301(2):619-26.

7. Witchel SF, Teede HJ, Peña AS. Curtailing PCOS. Pediatr Res. 2020;87(2):353-61

8. Osibogun O, Ogunmoroti O, Michos ED. Polycystic ovary syndrome and cardiometabolic 
risk: Opportunities for cardiovascular disease prevention. Trends Cardiovasc Med. 2020;30(7):399-404.

9. Deshpande PS, Gupta AS. Causes and prevalence of factors causing infertility in a public health facility. J Hum Reprod Sci. 2019;12(4):287-93

10. Rencber, S. F., Ozbek, S. K., Eraldemır, C., Sezer, Z., Kum, T., Ceylan, S., \& Guzel, E. (2018).
Effect of resveratrol and metformin on ovarian reserve and ultrastructure in PCOS: an experimental study. Journal of ovarian research, 11(1), 55.

11. Morgante G, Massaro MG, Di Sabatino A, Cappelli V, De Leo V. Therapeutic approach for metabolic disorders and infertility in women with PCOS. Gynecol Endocrinol. 2018;34(1):4-9. 